\title{
A Note on Reaction-Diffusion Systems with Skew-Gradient
}

\section{Structure}

\author{
Chao-Nien Chen* ${ }^{*}$ Tzyy-Leng Horng ${ }^{\dagger}$ Daniel Lee $^{\ddagger}$ Chen-Hsing Tsai ${ }^{\S}$
}

\section{Abstract}

Reaction-diffusion systems with skew-gradient structure can be viewed as a sort of activatorinhibitor systems. We use variational methods to study the existence of steady state solutions. Furthermore, there is a close relation between the stability of a steady state and its relative Morse index. Some numerical results will also be disussed.

${ }^{*}$ Department of Mathematics, National Changhua University of Education, Changhua Taiwan, ROC. Email : macnchen@cc.ncue.edu.tw

${ }^{\dagger}$ Department of Applied Mathematics, Feng Chia University, Taichung, Taiwan, ROC. E-mail : tlhorng@math.fcu.edu.tw

${ }^{\ddagger}$ Department of Mathematics, Tunghai University, Taichung, Taiwan, ROC. E-mail : danlee@thu.edu.tw

${ }^{\S}$ General Education Center, Chung Chou Institute of Technology, Changhua Taiwan, ROC. E-mail : jenshing@dragon.ccut.edu.tw

\section{Introduction}

In this note we consider reaction-diffusion systems of the form

$$
\begin{array}{cc}
M_{1} u_{t}= & D_{1} \Delta u+F_{1}(u, v), \\
M_{2} v_{t}= & D_{2} \Delta v-F_{2}(u, v), \\
& x \in \Omega, t>0 .
\end{array}
$$

Here $\Omega$ is a smooth bounded domain in $\mathbb{R}^{n}$, $u(x, t)$ is an $m_{1}$-dimensional vector function, $v(x, t)$ is an $m_{2}$-dimensional vector function, $M_{1}, M_{2}, D_{1}$ and $D_{2}$ are positive definite matrices, and there exists a function $F$ such that $\nabla F=\left(F_{1}, F_{2}\right)$. Such systems can be viewed as a sort of activator-inhibitor systems.

A well-known example is

$$
\begin{gathered}
u_{t}=d_{1} \Delta u+f(u)-v, \\
\tau v_{t}=d_{2} \Delta v+\sigma u-\gamma v
\end{gathered}
$$

where $d_{1}, d_{2}, \sigma, \gamma, \tau \in(0, \infty)$ and $f$ is a cubic polynomial. The case of $d_{2}=0$ has been con- 
sidered as a model for the Hodgkin-Huxley system $[13,22]$ to describe the behavior of electrical impulses in the axon of the squid. More recently, several variations of this system appeared in neural net models for short-term memory and in nerve cells of heart muscle.

As in [29], (1.1)-(1.2) will be referred as a skew-gradient system in which a steady state is a critical point of

$$
\begin{aligned}
\Phi(u, v)= & \int_{\Omega} \frac{1}{2}\left(D_{1} \nabla u, \nabla u\right)-\frac{1}{2}\left(D_{2} \nabla v, \nabla v\right) \\
& -F(u, v) d x
\end{aligned}
$$

A steady state $(\bar{u}, \bar{v})$ is called a mini-maximizer of $\Phi$ if $\bar{u}$ is a local minimizer of $\Phi(\cdot, \bar{v})$ and $\bar{v}$ is a local maximizer of $\Phi(\bar{u}, \cdot)$. It has been shown [29] that non-degenerate mini-maximizers of $\Phi$ are linearly stable. This result gives a natural generalization of a stability criterion for the gradient system in which all the nondegenerate local minimizers are stable steady states.

A remarkable property proved in [29] is that any mini-maximizer must be spatially homogeneous if $\Omega$ is a convex set. This kind of results have been established by Casten and Holland [5] and Matano [20] for the scalar reactiondiffusion equation, and generalized by Jimbo and Morita [15] and Lopes [19] for the gradient system. In case $\Omega$ is symmetric with respect to $x_{j}$, Lopes [19] showed that a global minimizer of gradient system is symmetric with respect to $x_{j}$; while Chen [7] obtained parallel results for the global mini-maximizers in the skew-gradient system.

In connection with calculus of variations, there is a close relation between the stability of a steady state of skew-gradient system and its relative Morse index. Based on this idea, some stability criteria for the steady states of (1.1)(1.2) are illustrated in section 2. In section 3, variational arguments are used to study the existence of steady states and their relative Morse indices. Section 4 contains numerical investigation of skew-gradient systems. A particular example to be studied is

$$
\begin{aligned}
u_{t} & =d_{1} u_{x x}+f(u)-v-w, \\
\tau_{2} v_{t} & =d_{2} v_{x x}+u-\gamma_{2} v, \\
\tau_{3} w_{t} & =d_{3} w_{x x}+u-\gamma_{3} w,
\end{aligned}
$$

which served as a model [4] for gas-discharge systems.

\section{Stability Criteria}

Let $E$ be a Hilbert space. For a closed subspace $U$ of $E, P_{U}$ denotes the orthogonal projection 
from $E$ to $U$ and $U^{\perp}$ denotes the orthogonal complement of $U$. For two closed subspaces $U$ and $W$ of $E$, denoted by $U \sim W$ if $P_{U}-P_{W}$ is a compact operator. In this case, both $W \cap U^{\perp}$ and $W^{\perp} \cap U$ are of finite dimensional. The relative dimension of $W$ with respect to $U$ is defined by

$$
\begin{aligned}
\operatorname{dim}(W, U)= & \operatorname{dim}\left(W \cap U^{\perp}\right) \\
& -\operatorname{dim}\left(W^{\perp} \cap U\right) .
\end{aligned}
$$

If $A$ is a self-adjoint Fredholm operator on $E$, there is a unique A-invariant orthogonal splitting

$$
E=E_{+}(A) \oplus E_{-}(A) \oplus E_{0}(A)
$$

with $E_{+}(A), E_{-}(A)$ and $E_{0}(A)$ being respectively the subspaces on which $A$ is positive definite, negative definite and null. For a pair of self-adjoint Fredholm operators $A$ and $\bar{A}$, it will be denoted by $A \sim \bar{A}$ if $E_{-}(A) \sim E_{-}(\bar{A})$. In this case, a relative Morse index $i(A, \bar{A})$ is defined by

$$
i(A, \bar{A})=\operatorname{dim}\left(E_{-}(\bar{A}), E_{-}(A)\right) .
$$

We refer to [1] for more details of relative Morse index.

For a critical point $(\bar{u}, \bar{v})$ of $\Phi$, let $\Phi^{\prime \prime}(\bar{u}, \bar{v})$ denote the second Frechet derivative of $\Phi$ at $(\bar{u}, \bar{v})$. A critical point $(\bar{u}, \bar{v})$ is called nondegenerate if the null space of $\Phi^{\prime \prime}(\bar{u}, \bar{v})$ is trivial. Let

$$
\begin{gathered}
M=\left(\begin{array}{cc}
M_{1} & 0 \\
0 & M_{2}
\end{array}\right), D=\left(\begin{array}{cc}
-D_{1} & 0 \\
0 & D_{2}
\end{array}\right), \\
Q=\left(\begin{array}{cc}
-I_{m_{1}} & 0 \\
0 & I_{m_{2}}
\end{array}\right)
\end{gathered}
$$

and $I_{k}$ be the $k \times k$ identity matrix. For a gradient system, it is known that a non-degenerate critical point with non-zero Morse index is an unstable steady state. The next theorem gives a parallel result for the skew-gradient system.

Theorem 1. Suppose $i\left(-Q, \Phi^{\prime \prime}(\bar{u}, \bar{v})\right) \neq 0$ and $\operatorname{dim} E_{0}\left(\Phi^{\prime \prime}(\bar{u}, \bar{v})\right)=0$, then for any positive definite matrices $M_{1}$ and $M_{2},(\bar{u}, \bar{v})$ is an unstable steady state of (1.1)-(1.2).

In [29] Yanagida pointed out an interesting property that a non- degenerate minimaximizer of $\Phi$ is always stable for any positive matrices $M_{1}$ and $M_{2}$ given in (1.1)-(1.2). An interesting question is whether there exist steady states with stability depending on the reaction rates of the system. Let $P^{+}$and $P^{-}$be the orthogonal projections from $E$ to $E_{+}(Q)$ and $E_{-}(Q)$ respectively. Define $\Psi_{0}=$ $M^{-\frac{1}{2}}\left(D \Delta-\nabla^{2} F(\bar{u}, \bar{v})\right) M^{-\frac{1}{2}}, \psi_{1}=P^{-} \Psi_{0} P^{-}$ 
and $\psi_{2}=P^{+} \Psi_{0} P^{+}$. Set $m=m_{1}+m_{2}, \quad W_{-}$, and $B_{r}=\{\xi \mid \xi \in E,\|\xi\|<r\}$. Assume $\mathfrak{D}=H^{2}\left(\Omega, \mathbb{R}^{m}\right)$,

that $\Phi(\xi)=\frac{1}{2}\langle\hat{\Lambda} \xi, \xi\rangle+b(\xi)$, where $\hat{\Lambda}$ is a self-

$$
\rho_{i}\left(\psi_{1}\right)=\inf _{z \in \mathfrak{D}} \frac{\left\langle\psi_{1} z, z\right\rangle_{L^{2}}}{\left\|P^{-} z\right\|_{L^{2}}^{2}}
$$

and

$$
\rho_{s}\left(\psi_{2}\right)=\sup _{z \in \mathfrak{D}} \frac{\left\langle\psi_{2} z, z\right\rangle_{L^{2}}}{\left\|P^{+} z\right\|_{L^{2}}^{2}}
$$

Theorem 2. Assume that $i\left(-Q, \Phi^{\prime \prime}(\bar{u}, \bar{v})\right)=0$ and $\operatorname{dim} E_{0}\left(\Phi^{\prime \prime}(\bar{u}, \bar{v})\right)=0$. Then $(\bar{u}, \bar{v})$ is stable if $\rho_{i}\left(\psi_{1}\right)>\rho_{s}\left(\psi_{2}\right)$.

Remark. In case we treat the Dirichlet adjoint invertible operator on $E, b \in C^{2}(E, \mathbb{R})$ and $b^{\prime}$ is compact. Set $S=\partial B_{\rho} \cap W_{+}$and $N=\left\{\xi^{-}+s e \mid \xi^{-} \in B_{r} \cap W_{-}\right.$and $\left.s \in[0, \bar{R}]\right\}$, where $e \in \partial B_{1} \cap W_{+}, r>0$ and $\bar{R}>\rho>0$. If $\Phi$ satisfies $(\mathrm{PS})^{*}$ condition and $\sup _{\partial N} \Phi<$ $\inf _{S} \Phi$, then $\Phi$ possesses a critical point $\bar{\xi}$ such that $\inf _{S} \Phi \geq \Phi(\bar{\xi}) \geq \sup _{\partial N} \Phi$. Moreover, if $W_{-} \sim E_{-}$, then

$$
\begin{aligned}
i\left(\hat{\Lambda}, \Phi^{\prime \prime}(\bar{\xi})\right) & \leq \operatorname{dim}\left(W_{-}, E_{-}\right)+1 \leq i\left(\hat{\Lambda}, \Phi^{\prime \prime}(\bar{\xi})\right) \\
& +\operatorname{dim} E_{0}\left(\Phi^{\prime \prime}(\bar{\xi})\right) .
\end{aligned}
$$

boundary condition $\left.u\right|_{\partial \Omega}=\left.v\right|_{\partial \Omega}=0, \mathfrak{D}$ is replaced by $H^{2}\left(\Omega, \mathbb{R}^{m}\right) \bigcap H_{0}^{1}\left(\Omega, \mathbb{R}^{m}\right)$.

The proofs of Theorem 1 and Theorem 2 can be found in [8].

Remark. (a) See e.g. [2, 8] for the definition of $(P S)^{*}$ condition.

(b) The index estimates (3.1) were obtained by Abbondandolo and Molina [2].

\section{Applications of Theorem 1 and Theorem 2}

In dealing with a strongly indefinite functional $\Phi$, a critical point theorem established by Benci and Rabinowitz [3] can be used to obtain steady states of (1.1)-(1.2).

$$
\begin{array}{r}
u_{t}=d_{1} \Delta u+f(u)-v, \\
\tau v_{t}=d_{2} \Delta v+u-\gamma v-h(v) .
\end{array}
$$

Theorem 3. Let $E$ be a separable Hilbert space with an orthogonal splitting $E=W_{+} \oplus$ A steady state of (3.2)-(3.3) is a critical point 
of

$\Phi(u, v)=\int_{\Omega} \frac{d_{1}}{2}|\nabla u|^{2}-\frac{d_{2}}{2}|\nabla v|^{2}-F(u, v) d x$,

where

$$
\begin{aligned}
F(u, v)= & -\left(\frac{1}{4} u^{4}-\frac{\beta+1}{3} u^{3}+\frac{\beta}{2} u^{2}\right)-u v \\
& +\frac{\gamma}{2} v^{2}+H(v),
\end{aligned}
$$

$\beta \in\left(0, \frac{1}{2}\right)$ and $H(v)=\int_{0}^{v} h(y) d y$. It is assumed that $\gamma>9\left(2 \beta^{2}-5 \beta+2\right)^{-1}$, and $h$ satisfies the following condition:

(h1) $h \in C^{1}, h(0)=h^{\prime}(0)=0$ and $y h(y) \geq 0$ for $y \in \mathbb{R}$.

Define

$$
\Lambda=\left(\begin{array}{cc}
-d_{1} \Delta-f^{\prime}(0) & 1 \\
1 & d_{2} \Delta-\gamma
\end{array}\right)
$$

Let

$$
\begin{aligned}
\mu_{k}^{+}= & \frac{1}{2}\left[\left(d_{1}-d_{2}\right) \lambda_{k}-\left(f^{\prime}(0)+\gamma\right)\right. \\
& \left.+\sqrt{\left(\left(d_{1}+d_{2}\right) \lambda_{k}-f^{\prime}(0)+\gamma\right)^{2}+4}\right]
\end{aligned}
$$

and

$$
\begin{aligned}
\mu_{k}^{-}= & \frac{1}{2}\left[\left(d_{1}-d_{2}\right) \lambda_{k}-\left(f^{\prime}(0)+\gamma\right)\right. \\
& \left.-\sqrt{\left(\left(d_{1}+d_{2}\right) \lambda_{k}-f^{\prime}(0)+\gamma\right)^{2}+4}\right]
\end{aligned}
$$

$\Lambda e_{k}^{+} \phi_{k}=\mu_{k}^{+} e_{k}^{+} \phi_{k}$ and $\Lambda e_{k}^{-} \phi_{k}=\mu_{k}^{-} e_{k}^{-} \phi_{k}$,

where

$$
\begin{aligned}
e_{k}^{+}= & \left(1, \frac{1}{2}\left[\sqrt{\left(\left(d_{1}+d_{2}\right) \lambda_{k}-f^{\prime}(0)+\gamma\right)^{2}+4}\right.\right. \\
& \left.\left.-\left[\left(d_{1}+d_{2}\right) \lambda_{k}-f^{\prime}(0)+\gamma\right]\right]\right), \\
e_{k}^{-}= & \left(1, \frac{-1}{2}\left[\left(d_{1}+d_{2}\right) \lambda_{k}-f^{\prime}(0)+\gamma\right.\right. \\
& \left.\left.+\sqrt{\left(\left(d_{1}+d_{2}\right) \lambda_{k}-f^{\prime}(0)+\gamma\right)^{2}+4}\right]\right) .
\end{aligned}
$$

It is clear that $\mu_{k}^{+}>0$ and $\mu_{k}^{-}<0$ for all $k \in \mathbb{N}$. Let $E_{+}=\oplus_{k=1}^{\infty} V_{k}^{+}$and $E_{-}=\oplus_{k=1}^{\infty} V_{k}^{-}$, where $V_{k}^{+}=\left\{s \phi_{k} e_{k}^{+} \mid s \in \mathbb{R}\right\}$ and $V_{k}^{-}=\left\{s \phi_{k} e_{k}^{-} \mid s \in\right.$ $\mathbb{R}\}$. Define $\Lambda^{+}=\left.\Lambda\right|_{E_{+}}, \Lambda^{-}=\left.\Lambda\right|_{E_{-}}$and

$$
\begin{aligned}
\left\langle\hat{\Lambda} z_{1}, z_{2}\right\rangle= & \int_{\Omega}\left(\left(\Lambda^{+}\right)^{\frac{1}{2}} z_{1},\left(\Lambda^{+}\right)^{\frac{1}{2}} z_{2}\right) \\
& -\left(\left(\Lambda^{-}\right)^{\frac{1}{2}} z_{1},\left(\Lambda^{-}\right)^{\frac{1}{2}} z_{2}\right) d x
\end{aligned}
$$

for $z_{1}, z_{2} \in E$. As an application of Theorem 3 , we have the following result.

Theorem 4. Let $\mathcal{B}_{R}$ be a ball in $\mathbb{R}^{n}$ with radius $R$. If $\Omega$ contains a ball $\mathcal{B}_{R}$ with $R$ being sufficiently large, then there exists a steady state $(\bar{u}, \bar{v})$ of $(3.2)-(3.3)$, and

$$
\begin{aligned}
& i\left(-Q, \Phi^{\prime \prime}(\bar{u}, \bar{v}) \leq 1 \leq i\left(-Q, \Phi^{\prime \prime}(\bar{u}, \bar{v})\right)\right. \\
& +\operatorname{dim} E_{0}\left(\Phi^{\prime \prime}(\bar{u}, \bar{v})\right)
\end{aligned}
$$

where $\left\{-\lambda_{k}\right\}$ are the eigenvalues of the Laplace operator and $\left\{\phi_{k}\right\}$ are the corresponding eigenfunctions. By straightforward calculation
In view of Theorem $1,(\bar{u}, \bar{v})$ is unstable if it is a non-degenerate critical point of $\Phi$. More details can be found in [8]. 
We now turn to some examples to seek stable there is a $j \in \mathbb{N}$ such that if steady states of skew-gradient systems. Consider

$$
\begin{aligned}
& d_{1} \lambda_{j}+\frac{1}{d_{2} \lambda_{j}+\gamma}<\alpha< \\
& \inf \left\{d_{1} \lambda_{k}+\frac{1}{d_{2} \lambda_{k}+\gamma} \mid k \in \mathbb{N} \backslash\{j\}\right\}
\end{aligned}
$$

$$
\tau v_{t}=\Delta v+2 v+u-|v| v \text {. }
$$

Straightforward calculation gives

$$
\Lambda=\left(\begin{array}{cc}
-\triangle+1 & 1 \\
1 & \Delta+2
\end{array}\right),
$$

$\mu_{k}^{+}=\frac{1}{2}\left(3+\sqrt{\left(2 \lambda_{k}-1\right)^{2}+4}\right)$ and $\mu_{k}^{-}=\frac{1}{2}(3-$ $\sqrt{\left.\left(2 \lambda_{k}-1\right)^{2}+4\right)}$. It is clear that $\mu_{k}^{+}>0$ for all $k \in \mathbb{N}$. Suppose $\Omega$ is a bounded domain in which the eigenvalue distribution of the Laplace operator (under homogeneous Dirichlet boundary conditions) satisfies the following property:

$$
\lambda_{1}<\frac{1}{2}(\sqrt{5}+1)<\lambda_{2} \leq \lambda_{3} \leq \cdots \leq \lambda_{k} \cdots
$$

Then it is easily seen that $\mu_{1}^{-}>0$, and $\mu_{k}^{-}<0$ if $k \geq 2$. It follows that $i(-Q, \Lambda)=-1$.

Theorem 5. There is a non-constant steady state $(\bar{u}, \bar{v})$ of (3.5)-(3.6). Moreover, if $\operatorname{dim}\left(\Phi^{\prime \prime}(\bar{u}, \bar{v})\right)=0$ and $\tau \geq \frac{2-\lambda_{1}}{1+\lambda_{1}}$, then $(\bar{u}, \bar{v})$ is stable.

In the next example, consider (1.3)-(1.4) with $f(u)=\alpha u-u^{3}$ and $\sigma=1$. Suppose

By direct calculation $\mu_{j}^{+}<0$ and $\mu_{k}^{+}>0$ for $k \in \mathbb{N} \backslash\{j\}$. Also, $\mu_{k}^{-}<0$ for all $k \in \mathbb{N}$. Hence $i(-Q, \Lambda)=1$. Applying Theorem 3 yields a steady state $(\bar{u}, \bar{v})$ of (1.3)-(1.4). Furthermore,

$$
\begin{aligned}
& i\left(-Q,-\Phi^{\prime \prime}(\bar{u}, \bar{v}) \leq 0 \leq i\left(-Q,-\Phi^{\prime \prime}(\bar{u}, \bar{v})\right)\right. \\
& +\operatorname{dim}_{0}\left(\Phi^{\prime \prime}(\bar{u}, \bar{v})\right) .
\end{aligned}
$$

This implies that $i\left(-Q,-\Phi^{\prime \prime}(\bar{u}, \bar{v})\right)=0$ if $(\bar{u}, \bar{v})$ is a non-degenerate critical point of $\Phi$. Then by Theorem 2, $(\bar{u}, \bar{v})$ is stable if $\tau<\frac{\gamma}{\alpha}$. In case of dealing with homogeneous Neumann boundary conditions, $(\bar{u}, \bar{v})$ is a spatially inhomogeneous steady state if (3.7) holds for $j \geq 2$. In other words, there exists a stable pattern for (1.3)(1.4).

For the FitzHugh-Nagumo system, the steady state solutions satisfy

$$
\begin{aligned}
d_{1} \Delta u+f(u)-v & =0, \\
\frac{d_{2}}{\sigma} \Delta v+u-\frac{\gamma}{\sigma} v & =0,
\end{aligned}
$$

where $f(u)=(1-u)(u-\beta) u, \beta \in\left(0, \frac{1}{2}\right)$. If $\mathcal{L}=$ $\sigma^{-1}\left(-d_{2} \Delta+\gamma\right)^{-1}$ under homogeneous Dirichlet (respectively Neumann) boundary conditions, 
then for any critical point $\bar{u}$ of

$\psi(u)=\int_{\Omega}\left[\frac{d_{1}}{2}\left(|\nabla u|^{2}+u \mathcal{L} u\right)-\int_{0}^{u} f(\zeta) d \zeta\right] d x$,

$(\bar{u}, \mathcal{L} \bar{u})$ is a steady state of FitzHugh-Nagumo system. In view of the fact that $\sigma \int_{\Omega} u \mathcal{L} u d x=$ $\int_{\Omega} d_{2}|\nabla v|^{2}+\gamma v^{2} d x$, it is easily seen that $\psi$ is bounded from below. In addition to minimizers, the Mountain Pass Lemma has been used to obtain non-trivial solutions $[9,10,11,17$, $21,24,28,32]$ of $(3.8)-(3.9)$

Let $u$ be a critical point of $\psi$. Straightforward calculation yields

$$
\psi^{\prime \prime}(u)=-\Delta+\mathcal{L}-f^{\prime}(u),
$$

where $\psi^{\prime \prime}$ is the second Frechet derivative of $\psi$ and the Morse index of $u$ will be denoted by $i_{*}\left(\psi^{\prime \prime}(u)\right)$. On the other hand, $(u, \mathcal{L} u)$ is also a critical point of

$$
\begin{aligned}
\Phi(u, v)= & \int_{\Omega}\left[\frac{d_{1}}{2}|\nabla u|^{2}-\frac{d_{2}}{2 \sigma}|\nabla u|^{2}+u v\right. \\
& \left.-\frac{\gamma}{2 \sigma} v^{2}-\int_{0}^{u} f(\xi) d \xi\right] d x .
\end{aligned}
$$

Proposition 1. If $u$ is a critical point of $\psi$ and $v=\mathcal{L} u$, then

$$
\operatorname{dim} E_{0}\left(\psi^{\prime \prime}(u)\right)=\operatorname{dim} E_{0}\left(\Phi^{\prime \prime}(u, v)\right)
$$

and

$$
i_{*}\left(\psi^{\prime \prime}(u)\right)=i\left(-Q, \Phi^{\prime \prime}(u, v)\right) .
$$

We refer to [8] for a proof of Proposition 1.

For a critical point $u$ obtained by the Mountain Pass Lemma, it is known [6] that

$$
i_{*}\left(\psi^{\prime \prime}(u)\right) \leq 1 \leq i_{*}\left(\psi^{\prime \prime}(u)\right)+\operatorname{dim} E_{0}\left(\psi^{\prime \prime}(u)\right) .
$$

Then by Proposition 1

$$
\begin{aligned}
& i\left(-Q, \Phi^{\prime \prime}(u, \mathcal{L} u)\right) \leq 1 \leq i\left(-Q, \Phi^{\prime \prime}(u, \mathcal{L} u)\right) \\
& +\operatorname{dim} E_{0}\left(\Phi^{\prime \prime}(u, \mathcal{L} u)\right) .
\end{aligned}
$$

Thus if $\operatorname{dim} E_{0}\left(\psi^{\prime \prime}(u)\right)=0$, it follows from Theorem 1 that $(u, \mathcal{L} u)$ is an unstable steady state of (1.3)-(1.4).

Let $\hat{\psi}_{1}=P^{-}\left(D \Delta-\nabla^{2} F(\bar{u}, \bar{v})\right) P^{-}, \hat{\psi}_{2}=$ $P^{+}\left(D \Delta-\nabla^{2} F(\bar{u}, \bar{v})\right) P^{+}$,

$$
\rho_{i}\left(\hat{\psi}_{1}\right)=\inf _{z \in \mathfrak{D}} \frac{\left\langle\hat{\psi}_{1} z, z\right\rangle_{L^{2}}}{\left\|P^{-} z\right\|_{L^{2}}^{2}}
$$

and

$$
\rho_{s}\left(\hat{\psi}_{2}\right)=\sup _{z \in \mathfrak{D}} \frac{\left\langle\hat{\psi}_{2} z, z\right\rangle_{L^{2}}}{\left\|P^{+} z\right\|_{L^{2}}^{2}} .
$$

Theorem 6. Assume that $i\left(-Q, \Phi^{\prime \prime}(\bar{u}, \bar{v})\right)=0$ and $\operatorname{dim} E_{0}\left(\Phi^{\prime \prime}(\bar{u}, \bar{v})\right)=0$. Then $(\bar{u}, \bar{v})$ is stable if one of the following conditions holds:

(i) $\rho_{i}\left(\hat{\psi}_{1}\right)>0, \rho_{s}\left(\hat{\psi}_{2}\right) \geq 0$ and

$$
\frac{\rho_{s}\left(\hat{\psi}_{2}\right)}{\rho_{i}\left(\hat{\psi}_{1}\right)}<\left\|M_{2}^{-1}\right\|^{-1}\left\|M_{1}\right\|^{-1} \text {. }
$$

(ii) $\rho_{i}\left(\hat{\psi}_{1}\right) \leq 0, \rho_{s}\left(\hat{\psi}_{2}\right)<0$ and

$$
\frac{\rho_{i}\left(\hat{\psi}_{1}\right)}{\rho_{s}\left(\hat{\psi}_{2}\right)}<\left\|M_{1}^{-1}\right\|^{-1}\left\|M_{2}\right\|^{-1} .
$$


Theorem 6 directly follows from Theorem 2 .

We refer to [8] for the detail. If $u$ is a non-degenerate minimizer of $\psi$ system :

and $v=\mathcal{L} u$, then Proposition 1 implies that $i(-Q, \Phi(u, v))=0$. Notice that

$$
\begin{array}{lc}
D \Delta-\nabla^{2} F(u, v)= & \\
\left(\begin{array}{cc}
-d_{1} \Delta-f^{\prime}(u) & 1 \\
1 & \frac{d_{2}}{\sigma} \Delta-\frac{\gamma}{\sigma}
\end{array}\right) .
\end{array}
$$

\section{1}

We start with the following reaction-diffusion

$$
\begin{aligned}
u_{t}= & d_{1} u_{x x}+u(u-\beta)(1-u) \\
& -v-w, \\
\tau_{2} v_{t}= & d_{2} v_{x x}+u-\gamma_{2} v, \\
\tau_{3} w_{t}= & d_{3} w_{x x}+u-\gamma_{3} w, \\
& x \in(0,1), t>0 .
\end{aligned}
$$

Since $f^{\prime}(\xi)=-3 \xi^{2}+2(\beta+1) \xi-\beta \leq\left(\beta^{2}-\right.$ where $\beta=0.3, \gamma_{2}=1, \gamma_{3}=20$, and the ho$\beta+1) / 3$, it easy to check that $\rho_{i}\left(\hat{\psi}_{1}\right)=$ mogeneous Neumann boundary conditions will $\rho_{i}\left(-d_{1} \Delta-f^{\prime}(u)\right) \geq d_{1} \lambda_{1}-\frac{\left(\beta^{2}-\beta+1\right)}{3}$ and be under consideration. In (4.1)-(4.3), u can $\rho_{s}\left(\hat{\psi}_{2}\right)=\rho_{s}\left(\frac{d_{2}}{\sigma} \Delta-\frac{\gamma}{\sigma}\right) \leq-\left(d_{2} \lambda_{1}+\gamma\right) / \sigma$, be viewed as an activator while $v$ and $w$ act where $\lambda_{1}<\lambda_{2} \leq \lambda_{3} \leq \cdots \leq \lambda_{k}<\cdots$ are as inhibitors. In view of the theoretical rethe eigenvalues of $-\Delta$. If $\rho_{i}\left(\hat{\psi}_{1}\right) \leq 0$ and sults mentioned in the previous sections, we $\tau<\frac{3\left(d_{2} \lambda_{1}+\gamma\right)}{\sigma\left(\left(\beta^{2}-\beta+1\right)-3 d_{1} \lambda_{1}\right)}$, condition (ii) of Theo- look for the pattern formation for (4.1)-(4.3) in rem 6 holds and consequently $(u, v)$ is a stable case the diffusion rate of the activator is small steady state of (1.3)-(1.4). $\quad\left(d_{1}=10^{-6}\right)$.

By taking $d_{2}=1$ and $d_{3}=10^{-6}$, various types of spatially inhomogeneous steady states have been observed through numerical calculation. In Figure 1 and Figure 3, there is one peak on the profile of $u$; the one in Figure 1 is symmetric with respect to the spatial variable, while the other is not. We found also instances of steady states with two peaks on the profile 
of $u$; but the distance between peaks can be different. We remark based on numerical observation that, with $\tau_{2}=\tau_{3}=10^{-4}$, such inhomogeneous steady states are stable under the flow generated by (4.1)-(4.3). Moreover, the solution profiles tell that $w$ is roughly equal to $\gamma_{3}^{-1} u$ in magnitude.

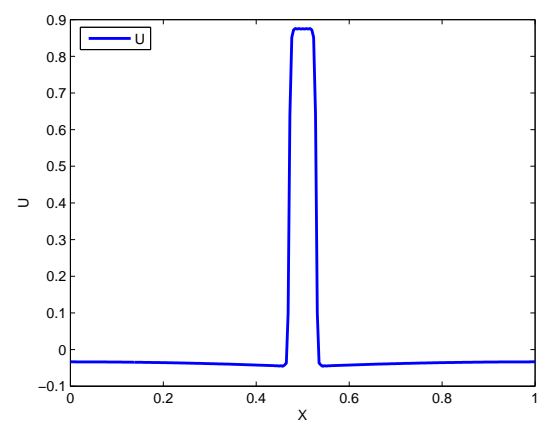

Figure 1: solution profile of $u$

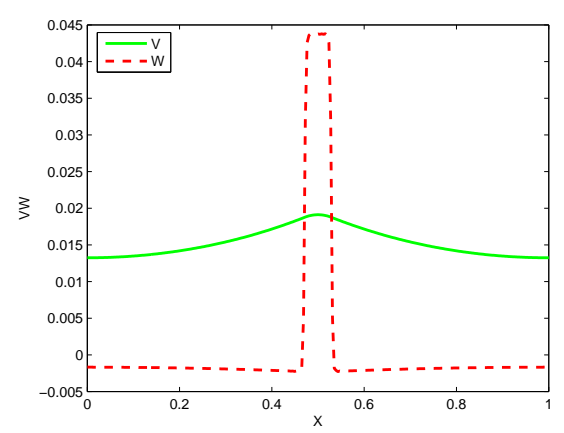

Figure 2: profiles of $v$ and $w$

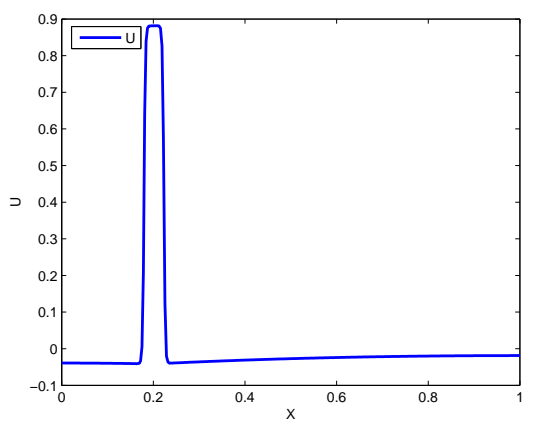

Figure 3: solution profile of $u$

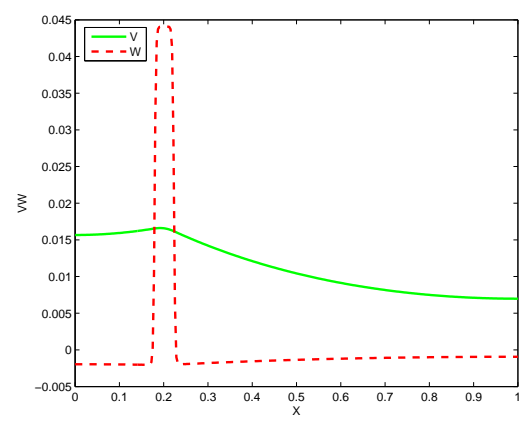

Figure 4: profiles of $v$ and $w$

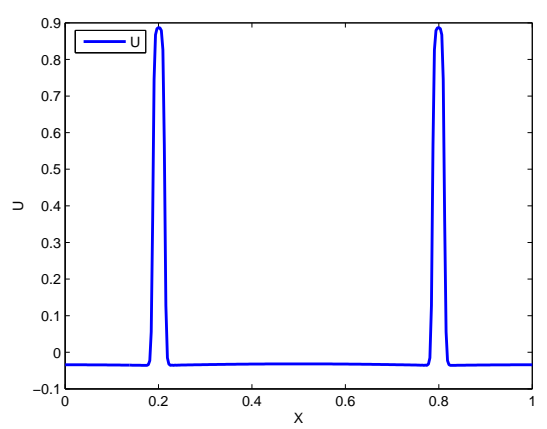

Figure 5: solution profile of $u$ 


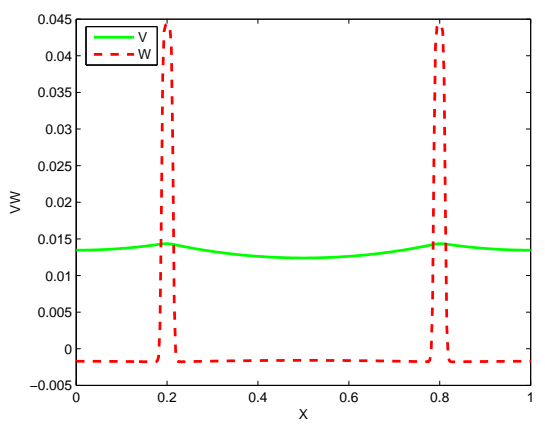

Figure 6: profiles of $v$ and $w$

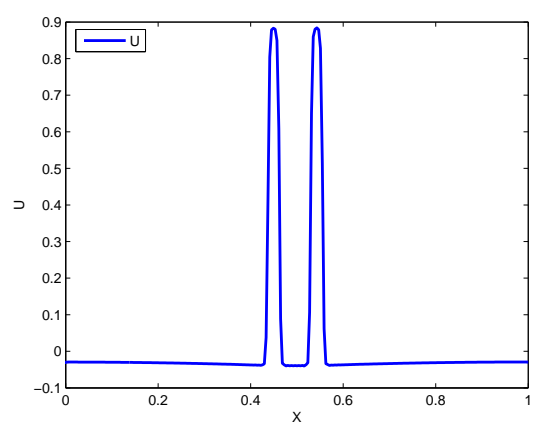

Figure 7: solution profile of $u$

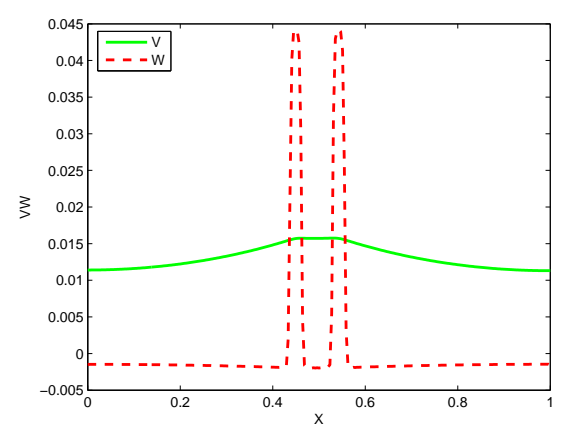

Figure 8: profiles of $v$ and $w$
We next turn to the case when both inhibitors $v$ and $w$ are acting with large diffusion $\left(d_{2}=d_{3}=1\right)$. As show in Figure 9-10, the pulse (or peak of $u$ ) becomes wider. The fact that $\gamma_{3}>\gamma_{2}$ results in $v>w$.

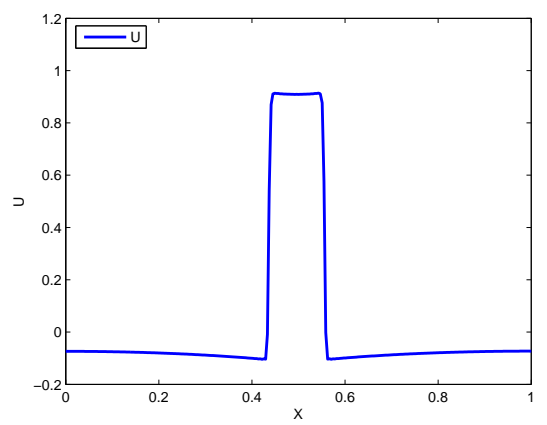

Figure 9: solution profile of $u$

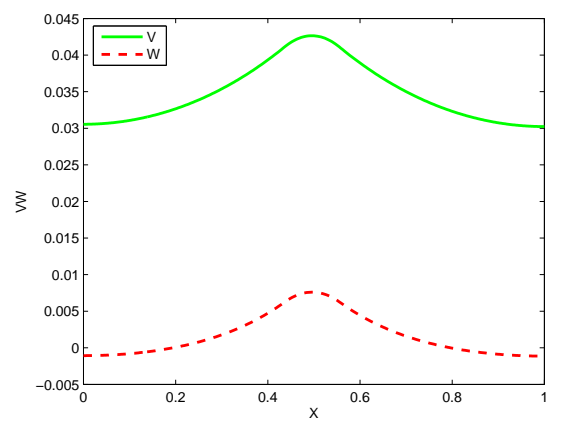

Figure 10: profiles of $v$ and $w$

Keeping $d_{3}=1$ and reducing $d_{2}$ to $10^{-1}$, we obtain a stable steady state with rather different profiles as shown in Figure 11-12. 


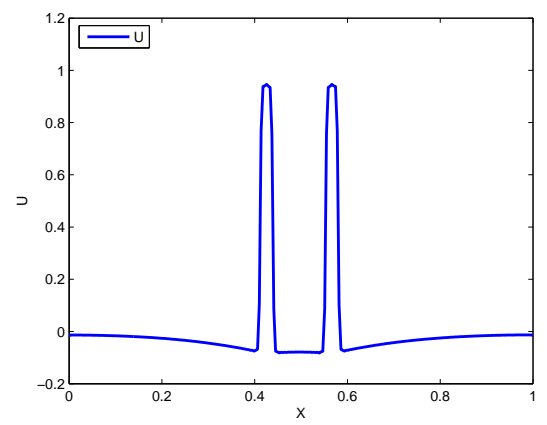

Figure 11: solution profile of $u$

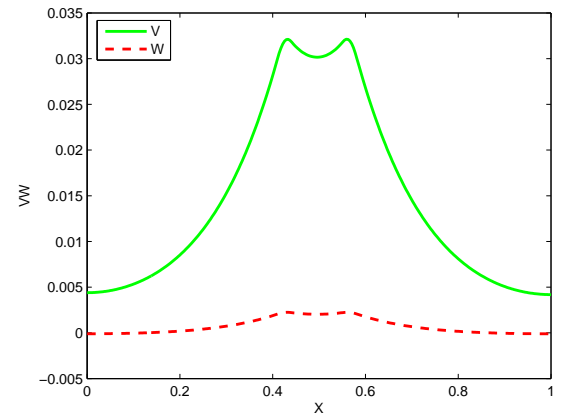

Figure 12: profiles of $v$ and $w$
As we know from Theorem 5, the choice of $\tau=0.1$ leads the flow converging to a nonconstant steady state (Figure 13). The behavior in the phase plane of the state variables, at the midpoint of the domain $(x=1.5)$, exhibits a spiral-inward convergence (Figure 14).

On the other hand, we conjecture that such a non-constant steady state become unstable if the value of $\tau$ is taking much smaller. Indeed, when $\tau=0.005$, we observed a time-periodic attractor (Figure 15-16) .

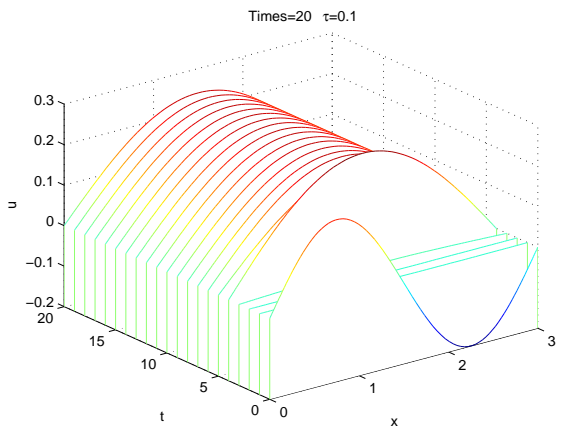

Figure 13: flow of $u$ with $\tau=0.1$

In this subsection we come back to the reaction-diffusion system

$$
\begin{aligned}
u_{t}= & u_{x x}-u-v, \\
\tau v_{t}= & v_{x x}+2 v+u-|v| v, \\
& x \in(0,3), t>0, \\
u(0, t)= & v(0, t)=u(3, t)=v(3, t)=0 .
\end{aligned}
$$

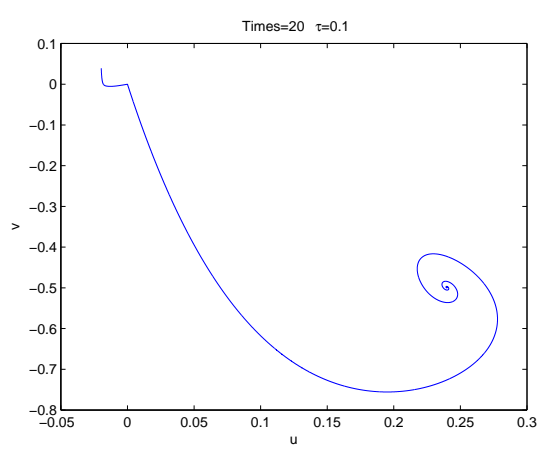


Figure 14: the trajectory of $(u(1.5, t), v(1.5, t))$
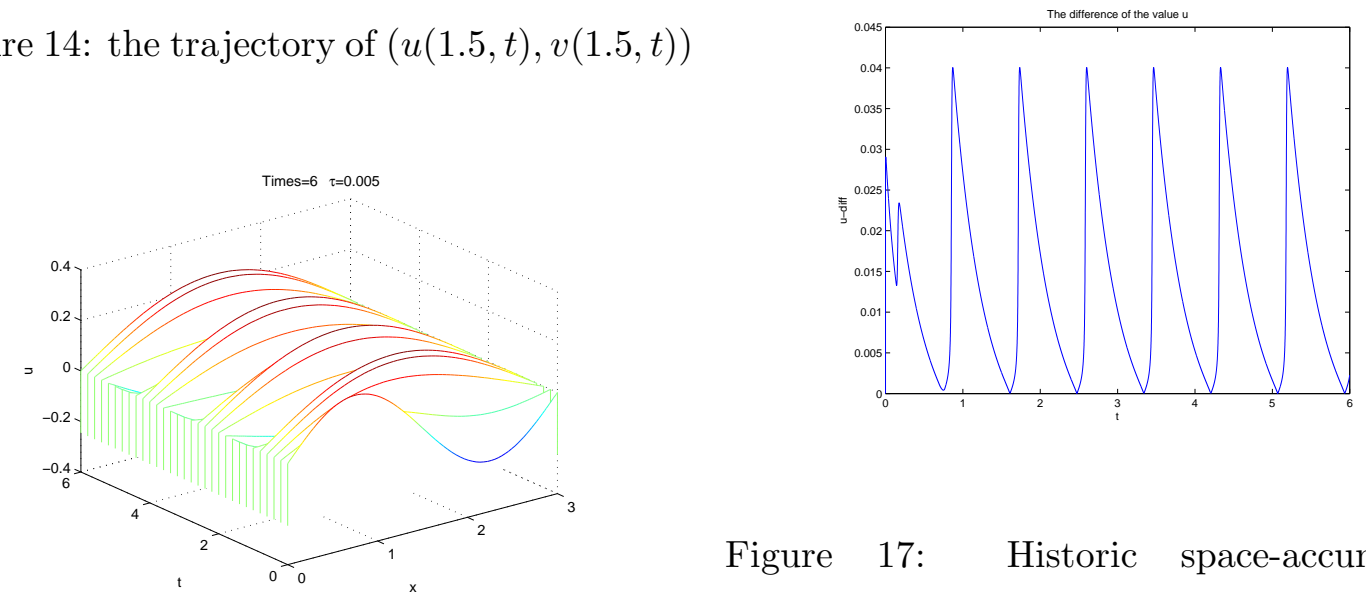

Figure 17: Historic space-accumulated $l_{1}$-difference of $u$

Figure 15: flow of $u$ with $\tau=0.005$
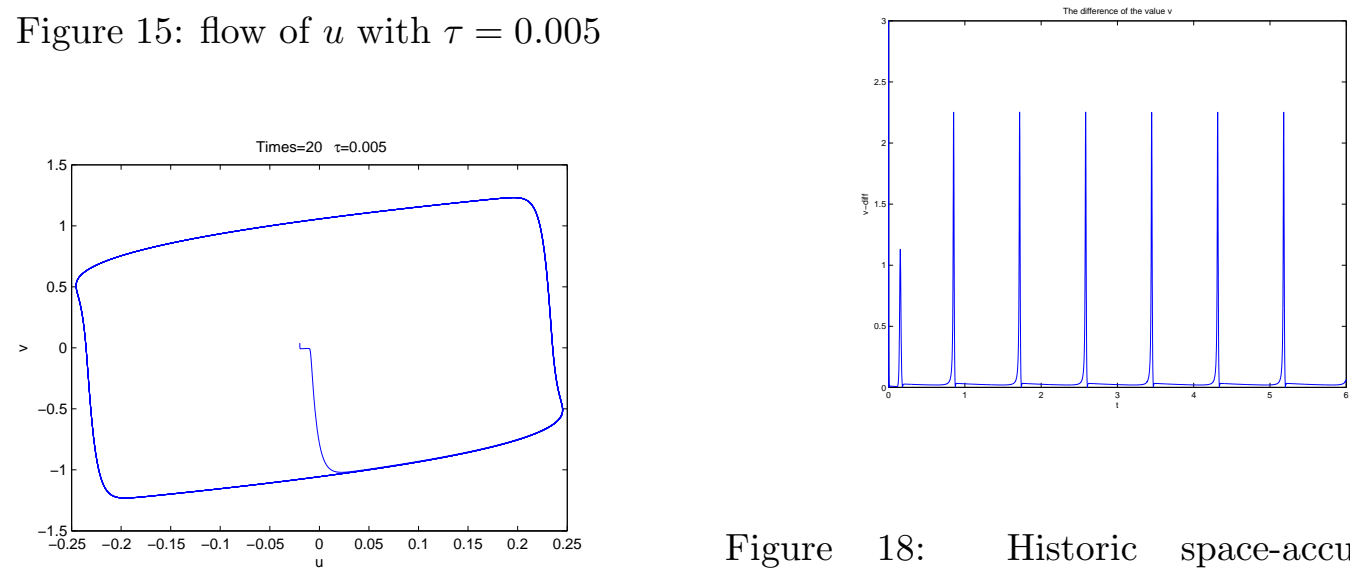

Figure 18: Historic space-accumulated $l_{1}$-difference of $v$

Figure 16: the trajectory of $(u(1.5, t), v(1.5, t)$

\section{Acknowledgments}

Research is supported in part by the Na-

The convergence history of the two calcutional Science Council, Taiwan, ROC. lated state variables is recorded in Figure 1718, which strongly suggests the existence of a stable time-periodic solution. The change of stability seems to result from a Hopf bifurcation and deserves further investigation.

\section{References}

[1] A. Abbondandolo, "Morse Theory for Hamiltonian Systems", Chapman 
Hall/CRC Research Notes in Mathematics, 425 (2001).

[2] A. Abbondandolo and J. Molina, Index estimates for strongly indefinite functionals, periodic orbits and homoclinic solutions of first order Hamiltonian systems, Calc. Var. 11 (2000), 395-430.

[3] V. Benci and P. H. Rabinowitz, Critical point theorems for indefinite functionals, Invent. Math. 52 (1979), 241-273.

[4] M. Bode, A. W. Liehr, C. P. Schenk and H. -G. Purwins, Interaction of dissipative solitons: particle-like behaviour of localized structures in a three-component reaction-diffusion system, Physica $D \mathbf{1 6 1}$ (2002), 45-66.

[5] R. G. Casten and C. J. Holland, Instability results for reaction diffusion equations with Neumann boundary conditions, J. Differential Equations 27 (1978), 266273.

[6] K. C. Chang, "Infinite Dimensioanl Morse Theory and Multiple Solution Problems", Birkhäuser. Basel, 1993.
[7] C. -C. Chen, Symmetry properties for the mini-maximizers of skew-gradient systems, Private communication.

[8] C. -N. Chen and X. Hu, Stability criteria for reaction-diffusion systems with skewgradient structure, Communications in Partial Differential Equations 33 (2008), 189-208.

[9] E. N. Dancer and S. Yan, Multipeak solutions for the Neumann problem of an elliptic system of FitzHugh-Nagumo type, Proc. London Math. Soc. 90 (2005), 209244.

[10] E. N. Dancer and S. Yan, A minimization problem associated with elliptic systems of FitzHugh-Nagumo type, Ann. IHPAnalyse Nonlineaire 21 (2004), 237-253.

[11] E. N. Dancer and S. Yan, Peak solutions for an elliptic system of FitzHughNagumo type, Ann. Sc. Norm. Super. Pisa Cl. Sci. 2 (2003), 679-709.

[12] D. G. de Figueiredo and E. Mitidieri, A maximum principle for an elliptic system and applications to semilinear problems, SIAM J. Math. Anal. 17 (1986), 836-849. 
[13] R. FitzHugh, Impulses and physiological states in theoretical models of nerve membrane, Biophys. J. 1 (1961), 445-466.

[14] A. Gierer and H. Meinhardt, A theory of biological pattern formation, Kybernetik 12 (1972), 30-39.

[15] S. Jimbo and Y. Mortia, Stability of nonconstant steady-state solutions to a Ginzburg-Landau equation in higher space dimensions, Nonlinear Anal. 22 (1984), 753-770.

[16] H. Kielhofer, Stability and semilinear evolution equations in Hilbert space, Arch. Rational Mech. Anal. 57 (1974), 150-165.

[17] G. Klaasen and E. Mitidieri, Standing wave solutions for system derived from the FitzHugh-Nagumo equations for nerve conduction, SIAM. J. Math. Anal. 17 (1986), 74-83.

[18] G. Klaasen and W. Troy, Stationary wave solutions of a system of reaction-diffusion equations derived from the FitzHughNagumo equations, SIAM J. Appl. Math. 44 (1984), 96-110.

[19] O. Lopes, Radial and nonradial minimizers for some radially symmetric function- als, Electron. J. Differential Equations 1996 (1996), 1-14.

[20] H. Matano, Asymptotic behaviour and stability of solutions of semilinear elliptic equations, Pub. Res. Inst. Math. Sci. 15 (1979), 401-454.

[21] H. Matsuzawa, Asymptotic profiles of variational solutions for a FitzHughNagumo type elliptic system, Differential Integral Equations 16 (2003) 897-926.

[22] J. Nagumo, S. Arimoto, and S. Yoshizawa, An active pulse transmission line simulating nerve axon, Proc. I. R. E. 50 (1962), 2061-2070.

[23] Y. Nishiura and M. Mimura, Layer oscillations in reaction-diffusion systems, SIAM J. Appl. Math. 49 (1989), 481-514.

[24] Y. Oshita, On stable nonconstant stationary solutions and mesoscopic patterns for FitzHugh-Nagumo equations in higher dimensions, J. Differential Equations 188 (2003), 110-134.

[25] P. H. Rabinowitz, "Minimax Methods in Critical Point Theory with Applications to Differential Equations", C.B.M.S. Reg. 
Conf. Series in Math. No. 65, Amer. Math. Soc., Providence, RI, 1986.

[26] C. Reinecke and G. Sweers, A positive solution on $\mathbb{R}^{n}$ to a equations of FitzHughNagumo type, J. Differential Equations 153 (1999), 292-312.

[27] C. Reinecke and G. Sweers, Existence and uniqueness of solutoin on bounded domains to a FitzHugh-Nagumo type elliptic system, Pacific J. Math. 197 (2001), 183211.

[28] X. Ren and J. Wei, Nucleation in the FitzHugh-Nagumo system: Interfacespike solutions, J. Differential Equations 209 (2005), 266-301.

[29] E. Yanagida, Mini-maximizers for reaction-diffusion systems with skewgradient structure, J. Differential Equations 179 (2002), 311-335.

[30] E. Yanagida, Standing pulse solutions in reaction-diffusion systems with skewgradient structure, J. Dynam. Differential Equations 14 (2002), 189-205.

[31] E. Yanagida, Reactoin-diffusion systems with skew-gradient structure, IMS
Workshop on Reaction-Diffusion Systems, Methods Appl. Anal. 8 (2001), 209-226.

[32] J. Wei and M. Winter, Clustered spots in the FitzHugh-Nagumo system, J. Differential Equations 213 (2005), 121-145. 